\title{
Analysis of the Electromagnetic Signature of Reinforced Concrete Structures for Nondestructive Evaluation of Corrosion Damage
}

\author{
Gemma Roqueta, Student Member, IEEE, Lluís Jofre, Fellow, IEEE, and Maria Q. Feng
}

\begin{abstract}
This paper presents a nondestructive corrosion damage detection method for reinforced concrete structures based on the analysis of the electromagnetic signature of the steel rebar corrosion. The signature of the corrosion on the scattered field upon microwave illumination is first numerically analyzed. First-order quality factor parameters, the energy and the mean propagation delay, are proposed to quantify the corrosion amount in the structure. To validate the model, low-profile ultra-wide-band antennas (3-12 GHz) are fabricated and measured. Measurements on 12 reinforced concrete samples with induced corrosion are performed, using three different antenna setups. The experiments demonstrate a good correlation between an increase in the corrosion amount with a decrease in the energy and an increase in the time delay of the propagated signal.
\end{abstract}

Index Terms-Corrosion, dielectric materials, microwave antennas, microwave imaging, microwave propagation, nondestructive testing, reflection, time domain analysis.

\section{INTRODUCTION}

$\mathbf{R}$ EINFORCED concrete is a major construction material for civil infrastructure, and the corrosion of steel reinforcing bars and tendons is its main cause of damage and early failure. Many failures of reinforced concrete structures in recent years, including the 2006 collapse of a concrete highway viaduct in Laval, Canada, and the resulting loss of five lives, remind us of the consequences for not dealing with corrosion problems in time [1]. A study conducted from 1999 to 2001 by the Federal Highway Administration [2] reveals that the direct cost of metallic corrosion to the U.S. economy is $\$ 276$ billion per year, which corresponds to approximately $2.5 \%$ of the U.S. gross domestic product in 2001. The size of the society challenge caused by corrosion is overwhelming. Early detection of corrosion would enable targeted and costeffective intervention, which is the key to saving tens of billions

Manuscript received April 26, 2011; revised August 8, 2011; accepted October 15, 2011. Date of publication November 22, 2011; date of current version March 9, 2012. This work was supported in part by the Spanish Interministerial Commission on Science and Technology (CICYT) through Projects TEC2007-66698-C04-01, TEC2010-20841-C04-02, and CONSOLIDER CSD2008-68, by the "Ministerio de Educación y Ciencia" through the Formación de Profesorado Universitario (FPU) fellowship program, and by the U.S. National Institute of Standards and Technology through the Technology Innovation Program. The Associate Editor coordinating the review process for this paper was Dr. Sergey Kharkovsky.

G. Roqueta and L. Jofre are with the Universitat Politècnica de Catalunya, 08034 Barcelona, Spain.

M. Q. Feng is with the Columbia University, New York, NY 10027-6902 USA.

Color versions of one or more of the figures in this paper are available online at http://ieeexplore.ieee.org.

Digital Object Identifier 10.1109/TIM.2011.2174106 of maintenance dollars and enhancing functionality and safety of the nation's civil infrastructure.

A number of electrochemical measurement techniques are available to assess the corrosion amount in reinforced concretes, including the half-cell potential [3] and the linear polarization resistance [4] techniques. Although these techniques are relatively easy to implement, even to scan an entire structure using modern probes, they might be impractical in certain situations since they often require partial exposure of steel reinforcement to connect a reference electrode. Additionally, there are some complicating factors owing to advances in concrete and repair technologies such as dense material overlays, corrosion inhibitors, chemical mixtures, and cathodic protection systems. Hence, it is important to consider to supplement the corrosion assessment with other nondestructive surveys.

Nondestructive evaluation (NDE) methods, which enable corrosion inspection from the surface of the concrete structure without requiring the partial exposure of the embedded rebar, are being intensively investigated in the recent years. Systems based on the ground penetrating radar (GPR) under traditional operation mode (100 MHz to $3 \mathrm{GHz}$ ) have been experimentally tested in [5] for the qualitative assessment of corrosion environment in the form of blurring of rebar image features due to the presence of moisture and chlorides in concrete. However, the operation principle and the common bandwidth used in traditional GPR systems may not provide sufficient resolution to quantify the corrosion rate. This reinforces the need of developing a comprehensive study of the electromagnetic reflections that take place inside concrete structures with corroded rebars. Thus, electromagnetic NDE technology can be improved for the early detection of steel corrosion, leading to significant cost saving and catastrophe prevention.

In this regard, an analysis of the electromagnetic signature of corroded steel reinforced concrete structures is performed in this study, aiming to reveal the most important requirements for the development of an optimized system capable of detecting and quantifying the steel corrosion at any stage of the corrosion process. In Section II of this paper, the electomagnetic modeling of a generic reinforced concrete structure with corrosion is first presented. A multilayer model to represent the effect of corrosion on reinforced concrete is developed. The frequency range for the electromagnetic illumination is then selected by taking into account the required resolution and penetrability. In Section III, the optimal measurable quality factors in the scattered field that capture most of the corrosion effects are described. The experimental validation is presented in Section IV, 
in which three types of experiments involving three different levels of complexity are performed. Finally, some conclusions are derived in Section V.

\section{Electromagnetic Modeling of a Reinforced CONCRETE STRUCture With CORRosion}

When illuminating concrete involving embedded steel rebar with a narrow pulse, the analysis of the propagated signal should provide information about the dielectric properties of the different components of the structure. In a corrosion process, electrochemical reactions between the concrete and the infiltrated oxygen and chloride lead to the formation of a rough layer of high dielectric material referred to as rust. This corrosion product represents a variation in the dielectric composition of the structure that may produce changes in the propagation of the electromagnetic waves through it.

In reality, penetration of moisture, salts, and other chemicals involved at the early stages of the corrosion process may significantly change dielectric properties of concrete as well. Concrete will also crack as corrosion progresses. However, for the sake of simplification, this study considers unchanged concrete used during testing. Nevertheless, such dielectric property variation or cracks in concrete could be as well used as an identification parameter for corrosion detection. This subject will be explored in the future.

This section is first devoted to the description of the chemical reactions that take place during the corrosion process. Then, based on these reactions and on the variations in the dielectric properties, the electromagnetic modeling of the corrosion coating is developed for the study of the scattering and the propagation through the structure.

\section{A. Corrosion Process}

Steel reinforced concrete, which is exposed to severe environmental agents such as sea water, rain, humidity, and deicing chemicals (salt), may experience a variation in the subsurface alkaline environment where the steel rebar is embedded. The chloride ion intrusion can disrupt the passive layer protecting the steel. Then, the electrochemical reactions between the water, oxygen, and the steel rebar lead to corrosion initiation and long-term progression to severe damage in concrete. Corrosion results in reduced cross-sectional area of the rebar and, thus, reduced strength capacity. Furthermore, the expanded rust layer causes concrete cracking and spalling. Aside from the electrochemical reactions to form corrosion on the steel, there are several criteria to describe the phases of the chlorideinduced corrosion process and the consequent deterioration of the concrete embedding the steel, which defines the service life of the concrete structures. This sort of classifications is very useful to optimize the NDE techniques depending on the corrosion stage of the structure. As pointed out in [6], a good classification can be derived from the combination of the diffusion-spalling model [7] and the simplified model of the concrete corrosion damage progression [8]. According to this classification, six phases of the concrete corrosion process can be targeted for nondestructive monitoring of the service life of a concrete structure, as depicted in Fig. 1.

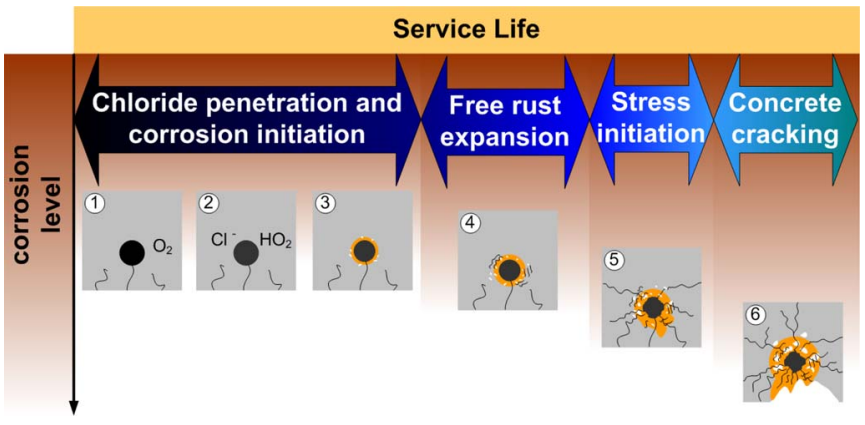

Fig. 1. Schematic illustration of the various steps in deterioration of the concrete embedding steel due to chloride-induced corrosion based on the classification done in [6].

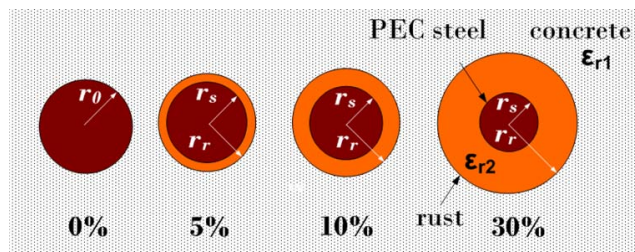

Fig. 2. Variation of the rebar and rust diameters for different corrosion amounts.

\section{B. Multilayer Model of the Corroded Reinforced Concrete Structure}

A multilayer dielectric model is considered to represent the effect of the corrosion on steel reinforced concrete. The corrosion amount on a rebar is typically calculated as the percentage of rebar weight loss with respect to a noncorroded rebar. Although the corrosion formation may not be uniform along the rebar, it is assumed that the loss of rebar weight is equivalent to a uniform loss of rebar volume. In addition, it has been reported in [9] that, when the volume of the rebar is reduced due to heavy corrosion, a volume of rust appears surrounding the rebar and occupying up to four times the corresponding loss of volume in the rebar. Then, the corrosion can be modeled as a uniform layer surrounding the steel rebar with intermediate conductivity and effective permittivity [10]. As a result, a three-layer model containing steel (conducting material), a rust coating (relative permittivity $\epsilon_{r 2}$ ), and the surrounding concrete (relative permittivity $\epsilon_{r 1}$ ), can be used to represent a corroded steel reinforced concrete structure. Fig. 2 graphically illustrates the decrease in steel rebar cross-sectional area and increase in the thickness of the rust layer as the corrosion amount increases. The higher the corrosion amount, the thicker the rust layer is and the smaller the rebar diameter is. The rebar corrosion amount $N$ is defined as the percentage of loss in rebar weight, i.e., volume. Thus, the final radius of the metallic rebar can be expressed as

$$
r_{s}=\sqrt{1-N} r_{0}
$$

where $r_{0}$ is the initial radius of the rebar. Then, the outer radius of the rust area can be written as

$$
r_{r}=\sqrt{1+3 N} r_{0}
$$




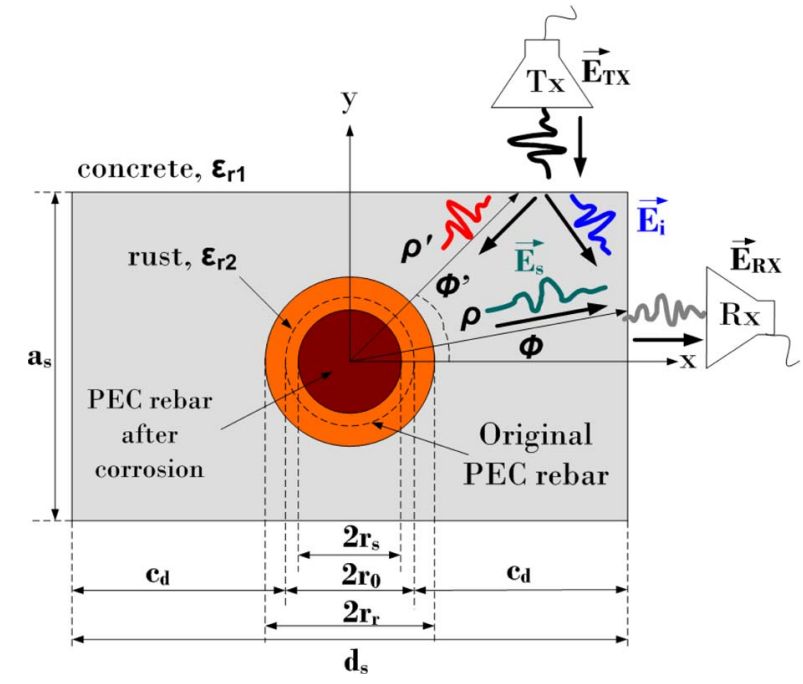

Fig. 3. Horizontal cut of the scenario: A transmitting antenna, placed at position $\vec{T}\left(\rho^{\prime}, \phi^{\prime}\right)$, illuminates with an electromagnetic field $\vec{E}_{t x}$ a scattering PEC cylinder (steel fiber) surrounded by a coating layer (rust) and embedded in a dielectric host medium (concrete specimen). The received field $\vec{E}_{r x}$ is captured by a receiving antenna placed at position $\vec{R}(\rho, \phi)$.

\section{Frequency Range Selection}

The selection of the appropriate frequency range for the illumination and visualization of the corrosion in steel reinforced concrete structures is a tradeoff between resolution and penetrability requirements, which are a function of the geometry of the concrete structure.

On the one hand, the frequency bandwidth $\left(B=f_{\max }-\right.$ $\left.f_{\text {min }}\right)$ is selected to guarantee a pulsewidth $(\Delta t=1 / B)$ that gives a range resolution $\left(\Delta x=c \Delta t / 2 \sqrt{\epsilon_{r 1}}\right)$, where the constant $c$ is the speed of light in free space. On the other hand, the maximum frequency $f_{\max }$ has to guarantee a good penetrability (capability to travel a total distance $d_{t}$ through the material under test) which is compatible with the dynamic range of the measurement equipment that is required.

In order to calculate the appropriate frequency range in this particular study, let us consider the rectangular scenario of Fig. 3, which is consistent with the concrete test samples in Fig. 5. The thickness of the concrete sample can be written as $d_{s}=2\left(c_{d}+r_{0}\right)$, which is assumed to be $10 \mathrm{~cm}$ in average. This average is calculated by considering two concrete cover depths $\left(c_{d}=3.75 \mathrm{~cm}\right.$ and $\left.c_{d}=5.00 \mathrm{~cm}\right)$ commonly seen in real concrete structures and a rebar diameter $2 r_{0}=12.5 \mathrm{~mm}$ (which is a \#4 rebar, according to the U.S. standards). The effective permittivity and attenuation of standard concrete are taken as $\epsilon_{r 1}=7$ and $\alpha=0.28 \mathrm{~dB} \cdot \mathrm{cm}^{-1} \cdot \mathrm{GHz}$ [11].

From the point of view of range resolution, in order to achieve a $\Delta x \leq 6 \mathrm{~mm}$, which can be considered appropriate to detect changes into the effective diameter of the rebar on the order of 1-2 mm, a pulsewidth $\Delta t \leq 0.1 \mathrm{~ns}$ is required. This leads to a frequency bandwidth $B \geq 9 \mathrm{GHz}$. Additionally, from the point of view of penetrability, the system has to guarantee a propagation penetrability $d_{t}$ better than $2 d_{s}=20 \mathrm{~cm}$ (corresponding to the round-trip propagation which is the case of largest propagation distance between transmitter and receiver). Taking into account conventional measurement equipment with a $0-\mathrm{dBm}$ transmitted power and a sensitivity of $-90 \mathrm{dBm}$, the total propagation losses have to be below this dynamic range (typically $70-80 \mathrm{~dB}$ ). Fulfilling these penetrability requirements, the maximum frequency chosen for the measurements is $f_{\max }=12 \mathrm{GHz}$. Therefore, the ultra wide band (UWB) frequency range from 3 to $12 \mathrm{GHz}$ can be considered for this application for its good compromise between resolution and penetrability. The capability of this frequency range will be first tested through numerical simulations in the next section.

\section{Numerical Simulation of the Scattering Principle}

The interaction between electromagnetic waves and a steel reinforced concrete structure is analyzed in order to identify quality factors for detecting the rebar corrosion. Fig. 3 shows a generic representation of the scenario to be considered in this model. A scattering perfect electric conductor (PEC) cylinder representing the remaining steel rebar is surrounded by a coating layer with relative permittivity $\epsilon_{r 2}$ representing the rust. A transmitter (Tx) at a position $\vec{T}\left(\rho^{\prime}, \phi^{\prime}\right)$ emits an electric field, $\vec{E}_{t x}$, which is transmitted to the structure. The reflections of this signal in the reinforced concrete structure, $\vec{E}_{r x}$, are captured by the receiver $(\mathrm{Rx})$ at position $\vec{R}(\rho, \phi)$. The structure is modeled as a multilayer geometry described in Fig. 2 .

Being in a first step, the steel corroded rebar is considered as an equivalent reduced-radius scattering cylinder embedded inside a dielectric medium, and the interaction of this kind of structures with the electromagnetic waves can be analyzed based on the formulation for scattering cylinders and cylindrical wave (infinite current filament) incidence [12]. This formulation considers the simplified model of a PEC cylinder with radius $r_{s}$ in (1) embedded in a medium with complex effective permittivity $\epsilon_{r 1}$. The total field at the receiver position may be expressed as the sum of the incident and the scattered field as

$$
\vec{E}_{r x}=\vec{E}_{s}+\vec{E}_{i}
$$

The incident field $\vec{E}_{i}$ can be understood as the existing field at the receiver position in the absence of the scattering cylinder. In turn, the field scattered by the cylinder $\left(\vec{E}_{s}\right)$ may be expressed as

$$
\begin{aligned}
\vec{E}_{s}= & \frac{k^{2} I}{4 \omega \epsilon_{r 1} \epsilon_{0}} \\
& \cdot \begin{cases}\sum_{n=-\infty}^{\infty} H_{n}^{2}\left(k \rho^{\prime}\right) \frac{J_{n}\left(k r_{s}\right)}{H_{n}^{2}\left(k r_{s}\right)} H_{n}^{2}(k \rho) e^{j \pi\left(\phi-\phi^{\prime}\right)} & \rho<\rho^{\prime} \\
\sum_{n=-\infty}^{\infty} H_{n}^{2}\left(k \rho^{\prime}\right) \frac{J_{n}\left(k r_{s}\right)}{H_{n}^{2}\left(k r_{s}\right)} H_{n}^{2}\left(k \rho^{\prime}\right) e^{j \pi\left(\phi-\phi^{\prime}\right)} & \rho>\rho^{\prime}\end{cases}
\end{aligned}
$$

where $H_{n}^{2}(x)$ is the Hankel function of the second kind, $J_{n}(x)$ is the Bessel function of the first kind, and $k=\omega / c \sqrt{\epsilon_{r 1}}$ is the phase constant. The constant $c$ is the velocity of propagation in free space.

The total received field is transformed to the time domain using the inverse Fourier transformation. The received pulse is then calculated as

$$
p_{r x}(t)=\frac{1}{2 \pi} \int_{0}^{\infty} E_{r x}(\omega) e^{j \omega t} d \omega .
$$


TABLE I

Steel Rebar $\left(2 r_{s}\right)$ AND RUSt DiAmeter $\left(2 r_{r}\right)$ FOR DifFERENT CORROSION AMOUNTS AND AN ORIGINAL STEEL DIAMETER $2 r_{0}=12,50 \mathrm{~mm}$

\begin{tabular}{|l|l|l|}
\hline Corrosion $(\%)$ & $2 r_{s}(\mathrm{~mm})$ & $2 r_{r}(\mathrm{~mm})$ \\
\hline \hline 0 & 12.50 & 12.50 \\
\hline 5 & 12.18 & 13.40 \\
\hline 10 & 11.85 & 14.25 \\
\hline 15 & 11.52 & 15.05 \\
\hline 20 & 11.18 & 15.81 \\
\hline 25 & 10.82 & 16.53 \\
\hline 30 & 10.45 & 17.23 \\
\hline
\end{tabular}

Using the time translation property of the Fourier transform [13], the delay of the propagated signal $\bar{\tau}$ can be related to the phase of the total electric field through

$$
\bar{\tau}=d_{m} \frac{\delta k}{\delta \omega}
$$

where $d_{m}$ is the distance of propagation.

Once it has been shown theoretically that the effect of the corrosion can be observed on the scattered field and, consequently, on the total received field as an equivalent reducedradius cylinder, a realistic model that considers not only a rebar diameter reduction [represented in (1)] but also the apparition of a rust layer [represented in (2)] is considered for simulations. A finite-difference time-domain (FDTD) code (MEEP from Massachusetts Institute of Technology [14]) is used to simulate the interaction between electromagnetic waves and a multilayer structure in a lossy dielectric medium, that represents a reinforced concrete structure with corroded steel rebar. A transmitter (Tx) and a receiver ( $\mathrm{Rx})$ are placed at positions $\vec{T}\left(\rho^{\prime}=c_{d}=50.0 \mathrm{~mm}, \phi^{\prime}=0\right)$ and $\vec{R}\left(\rho=c_{d}=50.0 \mathrm{~mm}\right.$, $\phi=\pi)$, respectively. A scenario with a conducting cylinder of initial diameter $2 r_{0}=2 r_{s}=12.5 \mathrm{~mm}$ embedded in a concrete dielectric medium of effective permittivity $\epsilon_{r 1}=4-\mathrm{j} 0.06$ (approximation derived from [15] and [16]) is considered for the simulations. As reported in [17], a realistic value for the effective permittivity of the rust layer can be $\epsilon_{r 2}=10-\mathrm{j} 0.86$. Corrosion amounts from $0 \%$ to $30 \%$ in steps of $5 \%$ increase are considered in the simulations. Table I lists the diameter of the conducting cylinder $r_{s}$ and the rust layer $r_{r}$ for each corrosion amount, calculated according to (1) and (2).

The total received pulse for each corrosion amount is calculated according to (5) and represented in Fig. 4. From now on, results will be always normalized with respect to the reference with $0 \%$ rust. Results show that the selected UWB frequency range $(3-12 \mathrm{GHz})$ allows the generation of narrow pulses, so differences in the received signal due to rust generation can be detected with reasonable levels of attenuation. Notice that the presence of rust represents an increase of the attenuation and the propagation delay of the received signal in all cases. The increase of the propagation delay in the corroded rebar may be explained as duel consequences: On one side, the reduction of the rebar diameter implies a reduction of the electrical distance for the waves to propagate, and on the other side, the presence of an extra intermediate permittivity dielectric material (corrosion) surrounding the rebar makes this path longer. As a result, the propagation delay increases with respect to the noncorroded rebar (or equivalently, the net propagation speed decreases).

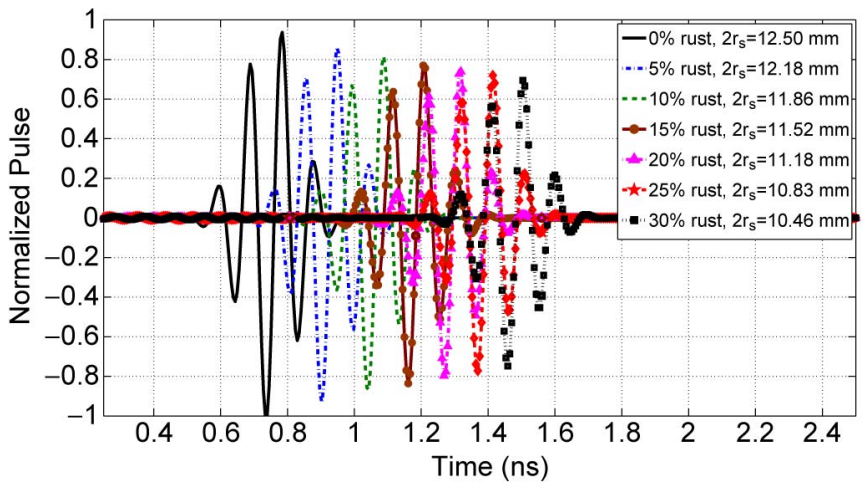

Fig. 4. FDTD simulation of total received pulse at the receiver position $\vec{R}\left(\rho=c_{d}=50 \mathrm{~mm}, \phi=\pi\right)$ in a scenario with a PEC scattering cylinder of radius $r_{s}$ that varies as a function of the corrosion amount $(0 \%-30 \%)$. The pulses are normalized with respect to the reference simulation with $0 \%$ rust.

\section{First-Order Quality FACtors FOR CORROSION DETECTION}

In order to test the hypothesis that the effect of the corrosion in the steel rebar embedded in concrete structures can be observed in the scattered field by a microwave illumination through real measurements, it is important to identify the optimal measurable first-order quality factors in the scattered field that capture the most information of the corrosion effects. The corrosion can be represented as a linear system with an impulse response $h(t)$ that relates the received pulse $p_{r x}(t)$ to the transmitted pulse $p_{t x}(t)$ through

$$
p_{r x}(t)=p_{t x}(t) * h(t) .
$$

Applying the Fourier transform property of the convolution and rearranging the equation above, it is derived that $H(\omega)=$ $E_{r x}(\omega) / E_{t x}(\omega)$, where $H(\omega)$ is defined as the transfer function and contains the information of the corrosion in the structure in the frequency domain. In practice, signals are obtained in the frequency domain and then Fourier transformed into the time domain for an easier calculation of the impulse response.

In the case of a structure embedding a high permittivity target with an electrically smooth surface (roughness small compared to the wavelength) such as the cylindrical steel rebar described earlier, a portion of the electromagnetic waves may travel guided through the cylinder surface. These may be seen as traveling creeping waves, whose return comes from behind the rebar. Due to the layer of the rust cover on the steel rebar, the creeping waves gather most of the corrosion effects and represent the main variations on the scattered field observed in the FDTD simulations shown in Fig. 4, mainly the delay and the attenuation.

In practice, the analysis and identification of every individual reflection can be highly challenging. However, it has been shown in [18] that the combination of all the reflections is well represented in the energy and the mean propagation delay of the total reflected signal. The amount of energy contained in the reflected waves can be related to the impulse response of the system $h(t)$ as [19]

$$
\mathcal{E}=\int_{0}^{\infty}\left|h\left(\tau^{\prime}\right)\right|^{2} d \tau^{\prime} .
$$




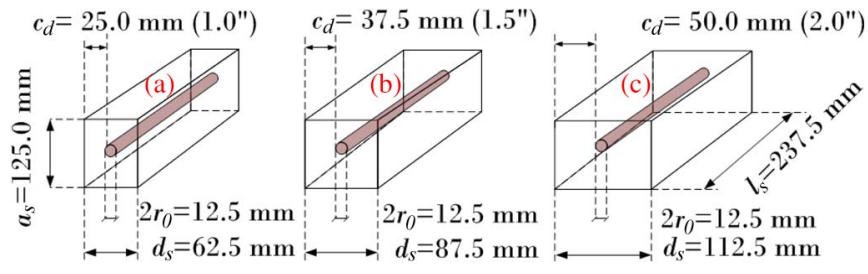

Fig. 5. Scheme of the three types of blocks used in this experiment with different cover depths. (a) $25.0 \mathrm{~mm}\left(1.0^{\prime \prime}\right)$. (b) $37.5 \mathrm{~mm}\left(1.5^{\prime \prime}\right)$. (c) $50.0 \mathrm{~mm}$ $\left(2.0^{\prime \prime}\right)$.

Then, the mean propagation delay may be expressed as

$$
\bar{\tau}=\frac{\int_{0}^{\infty} \tau^{\prime}\left|h\left(\tau^{\prime}\right)\right|^{2} d \tau^{\prime}}{\mathcal{E}} .
$$

Therefore, the energy and the mean propagation delay are considered to be the basic first-order quality factors of the scattered field that contain the most information about the rebar corrosion in the structure.

\section{EXPERIMENTAL VALIDATION}

Once the electromagnetic model of the corroded rebar embedded in concrete structures has been developed and the firstorder quality factors revealing corrosion information from the scattered field have been identified, an experimental validation on a set of reinforced concrete specimens with different corrosion rates is then performed.

\section{A. Samples Under Test}

In total, 12 reinforced concrete samples, each one embedding a single \#4 rebar, are fabricated and subjected to a controlled accelerated corrosion process. The ready-to-use concrete mix, which contains cement, sand, and coarse aggregates, was used to fabricate the concrete specimens. In order to test the resolution and penetrability performance of the measurement system, the study is focused on three of the reinforced concrete samples with different concrete cover depths and a rebar diameter of $2 r_{0}=12.5 \mathrm{~mm}$. Fig. 5 shows a scheme of these samples. Please note that the authors use the U.S. customary unit inches within parenthesis to refer to the cover depth $c_{d}$ to be consistent with the standard U.S. terminology. Samples of type A have a cover depth of $c_{d}=25.0 \mathrm{~mm}\left(1.0^{\prime \prime}\right)$ and a total width of $d_{s}=62.5 \mathrm{~mm}$. Samples of type B have a cover depth of $c_{d}=$ $37.5 \mathrm{~mm}\left(1.5^{\prime \prime}\right)$ and a total width of $d_{s}=87.5 \mathrm{~mm}$. Finally, samples of type $\mathrm{C}$ have a cover depth of $c_{d}=50.0 \mathrm{~mm}\left(2.0^{\prime \prime}\right)$ and a total width of $d_{s}=112.5 \mathrm{~mm}$.

Four different corrosion amounts covering the different phases of the corrosion process, $0 \%$ (phase 1), $5 \%$ (phase 2-3), $10 \%$ (phase 4-5), and 30\% (phase 6), are introduced to the samples using the impressed current method [20]. It is noted that the microwave measurements (to be presented as follows) were taken on these samples three months after their accelerated corrosion process, when the samples were fully air-dried in an air-conditioned laboratory. A photography of the samples used for the tests is shown in Fig. 6.

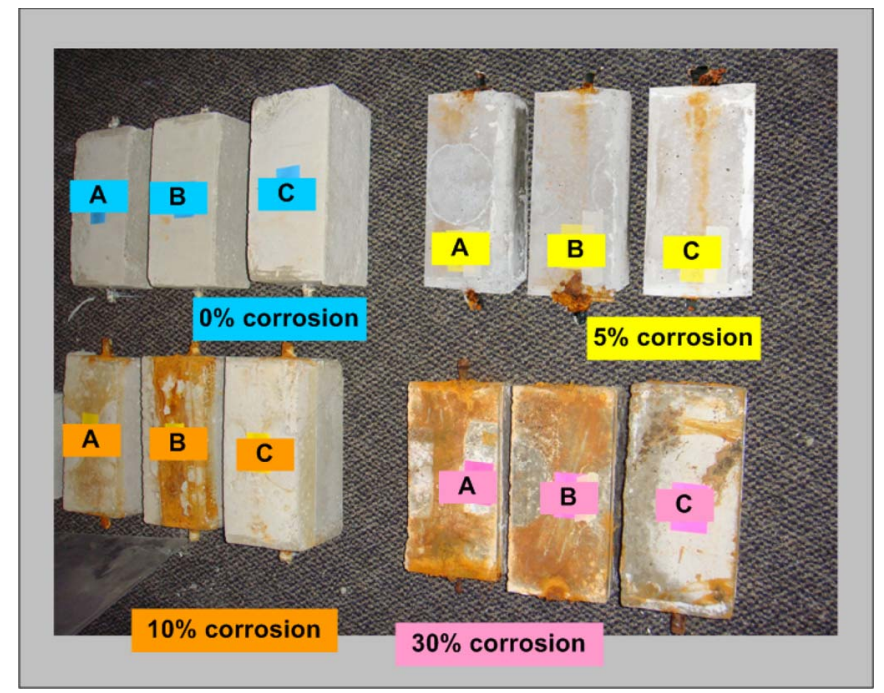

Fig. 6. Photography of test specimens and corroded rebars used in the tests.

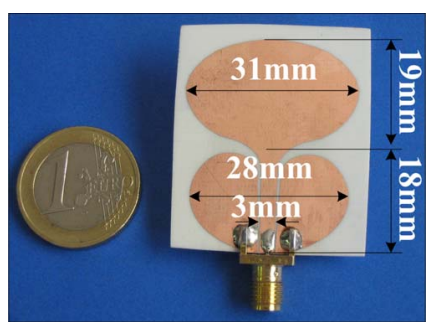

Fig. 7. Photograph of the UWB elliptical monopole antenna (3-12 GHz) used in the measurements for the characterization of the corrosion in steel reinforced concrete structures.

\section{B. Measurement System}

A network analyzer, Agilent $8722 \mathrm{ES}(50 \mathrm{MHz}$ to $40 \mathrm{GHz}$ ), is used for the generation of the excitation signal as well as for the acquisition of the frequency-domain measured signals in the selected frequency range of $3-12 \mathrm{GHz}$. These signals are the four combinations of the $\mathrm{S}$ parameters $\left(\mathrm{S}_{i i}\right.$ reflection and $\mathrm{S}_{i j}$ transmission).

UWB (3-12 GHz) planar elliptical monopole antennas [21] are fabricated with a bended ground plane and fed by a coplanar waveguide. The antenna shape and dimensions are shown in Fig. 7. These commonly used UWB antennas provide a good matching that varies gently over the bandwidth of interest, and their horizontal radiation pattern is almost omnidirectional at all frequencies. This is translated into a narrow-band pulse in the time domain with low ripple level. Also, the compact size and ease of handling make this type of antenna particularly ideal for in situ inspection of concrete structures. For the sake of maximizing the sensitivity to corrosion, the antennas are placed in such a way that their direction of polarization is always parallel to the orientation of the steel rebar. The antennas are placed at few centimeters away from the concrete surface, so the reflection at the first interface (air-concrete) is well identified and removed from the signal of interest by means of postprocessing gating.

Three types of antenna setups, as shown in Fig. 8, are tested to determine the best scenario for corrosion characterization. First, Fig. 8(a) shows a through-transmission setup in which 


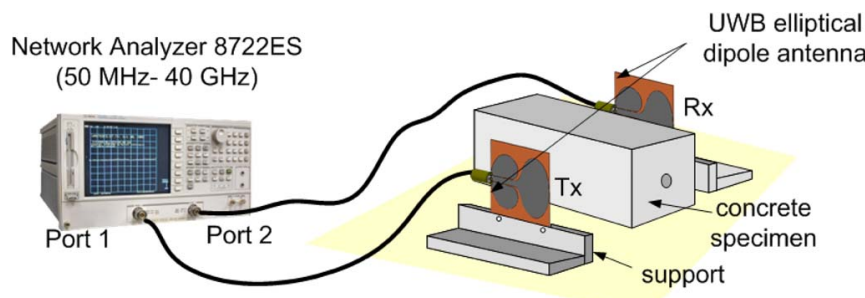

(a)

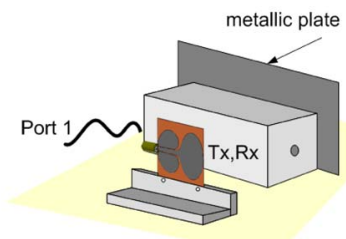

(b)

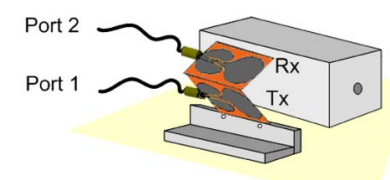

(c)

Fig. 8. Scheme of the measurement setup with a network analyzer and the three types of setup: (a) Through-transmission, (b) monostatic-reflection, and (c) bistatic setups.

a transmitter is placed at position $\vec{T}\left(\rho^{\prime}=c_{d}, \phi=0\right)$ and a receiver is placed at $\vec{R}\left(\rho=c_{d}, \phi=\pi\right)$. Fig. 8(b) illustrates a monostatic-reflection setup in which a single antenna placed at $\left(\rho=c_{d}, \phi=0\right)$ acts both as a transmitter and a receiver and a metallic plate is placed at the end of the structure for calibration purposes. Finally, Fig. 8(c) shows a bistatic setup, in which a transmitter and a receiver are placed at $\left(\rho=c_{d}, \phi=0\right)$ forming an angle of $120^{\circ}$ between them. This angle corresponds to the required inclination between the antennas so that their main beams collide at the center of the concrete structure.

Depending on the measurement setup, the interest is focused on the acquisition of the transmission [see Fig. 8(a) and (c)] or the reflection [see Fig. 8(b)] S parameters. In all the experiments, the $\mathrm{S}$ parameter corresponding to the signal of interest, $H(\omega)=S_{\text {port }_{r x} / \text { port }_{t x}}=E_{r x}(\omega) / E_{t x}(\omega)$, is transformed to the impulse response, $h(t)$, using the inverse Fourier transform. In practice, the limits of the integral are determined by the frequency range of measurements, so $H(\omega)$ is implicitly multiplied by a rectangular window that generates high secondary lobes in the inverse transform. In order to obtain a smooth inverse transform with low level secondary lobes, $H(\omega)$ is filtered using a Gaussian window ( $\sigma=1 \mathrm{~ns})$. Additionally, once $h(t)$ is obtained, time gating is applied in order to remove the direct coupling between the antennas.

\section{Experiment 1: Through-Transmission Setup}

A through-transmission setup such as the one in Fig. 8(a) is chosen as a proof-of-concept test to validate the numerical simulations of the scattering principle presented in Section II-C. Such a setup allows easy analysis of the measured signals, although it may not be practical for in situ inspection of concrete structures. In this setup, the interest is focused on the transmission parameter $\mathrm{S}_{12}\left(=\mathrm{S}_{21}\right)$. The transfer function $H(\omega)=S_{12}(\omega)$ is Fourier transformed to the time domain, and the resulting impulse responses are shown in Fig. 9 for the signals measured on all 12 reinforced concrete samples with 3 different concrete cover depths and 4 different corrosion amounts. A progressive displacement and attenuation of the

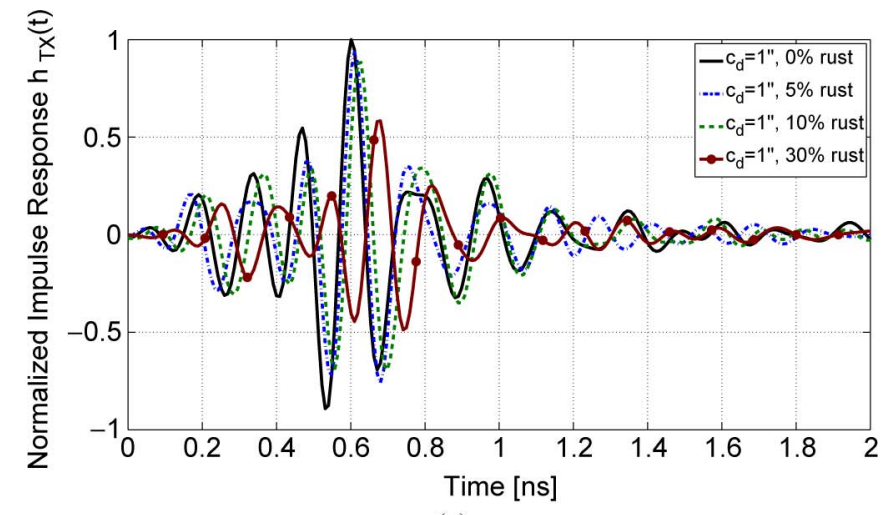

(a)

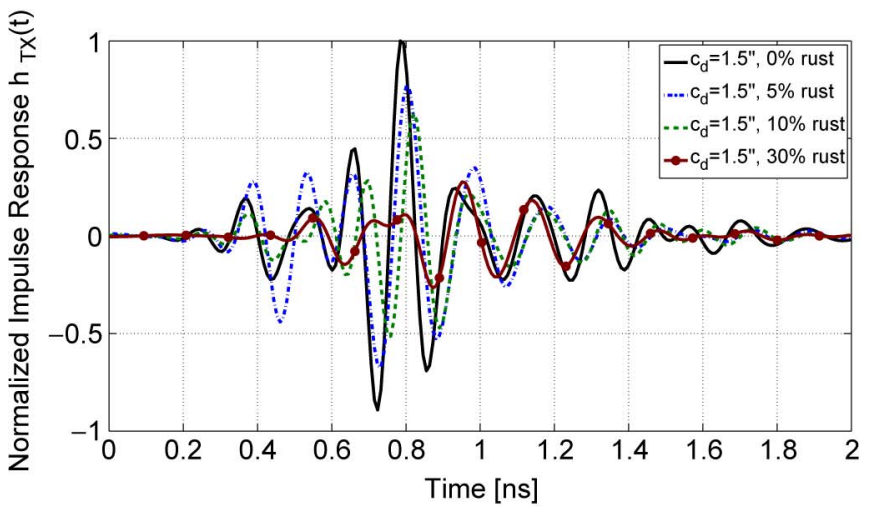

(b)

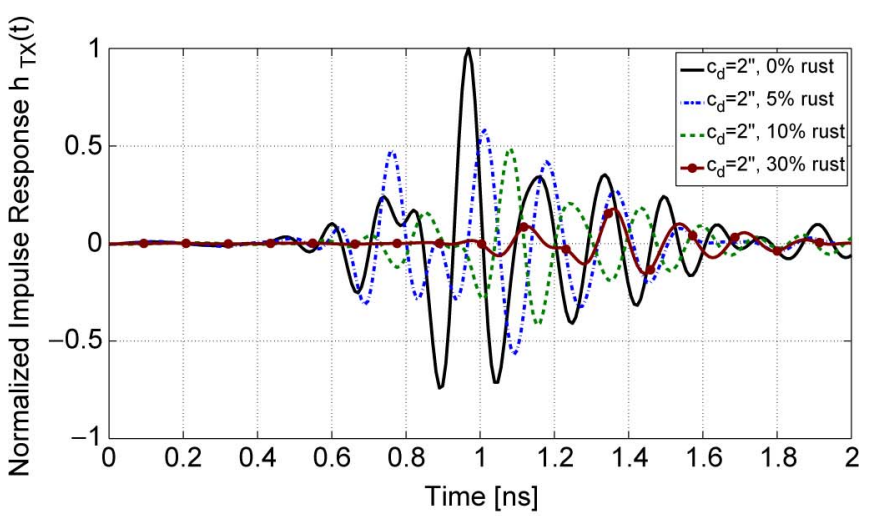

(c)

Fig. 9. Measured impulse response $h(t)$ normalized with respect to the noncorroded measurement, using a through-transmission setup for (a) cover depth A, (b) cover depth B, and (c) cover depth C, for each corrosion amount from $0 \%$ to $30 \%$.

main peak are observed in the experimental results presented in this figure, which is consistent with the analytical results in Fig. 4. When comparing the different cover depths (A, B, and $\mathrm{C}$ defined in Fig. 5) in Fig. 9(a)-(c), it is observed that the thicker the cover depth, the faster (and more visible) the phase and attenuation variations due to corrosion are. This may be caused by the better capability of the system to distinguish the different reflections in a thick concrete cover. In such a case, the propagation distance is larger than the pulsewidth, which reduces the main pulse distortion due to a too close reflecting corroded rebar. Additionally, this may also be due to nonlinear increases in signal attenuation due to scattering and spherical spreading of the electromagnetic waves, which would be more prominent at increased cover depths. 


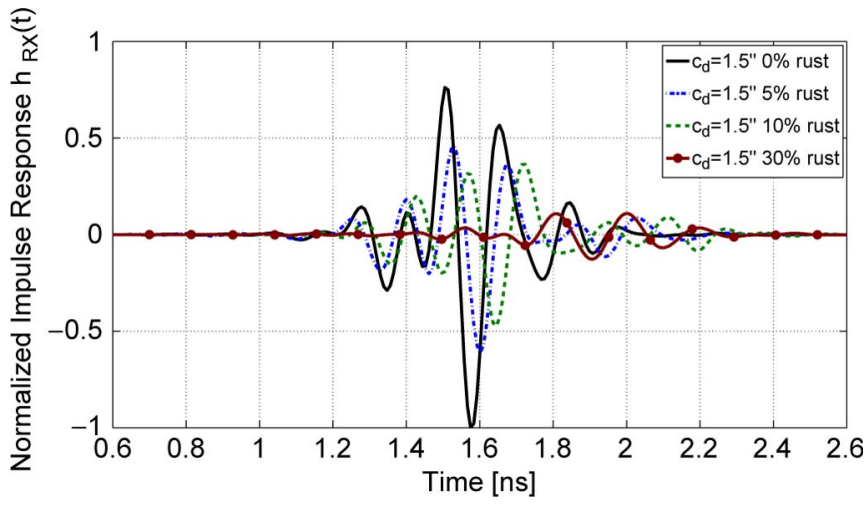

Fig. 10. Measured impulse response $h(t)$ normalized with respect to the noncorroded measurement, using a monostatic-reflection setup for the cover depth B and all the corrosion amounts from $0 \%$ to $30 \%$.

\section{Experiment 2: Monostatic-Reflection Setup}

While the through-transmission setup is used as the first step to prove the concept of the microwave visualization of corrosion, the monostatic-reflection setup is ideal for practical applications in in situ inspection of concrete structures. In this setup, the interest is focused on the reflection parameter $\mathrm{S}_{11}(=$ $\mathrm{S}_{22}$ ). The overall signal propagates through the structure and is reflected at its back wall. Since the propagation distance (round trip) is twice the distance propagated in the throughtransmission setup, the received pulse is expected to be weak and contains the combination of multiple interferences. In order to improve the signal-to-noise ratio, a differential calibration approach is proposed. The idea is to combine a measurement of the scenario with a high reflectivity material (such as a metal plate) placed behind the concrete structure and a measurement with a different permittivity material (without the metal plate). This differential calibration could be naturally performed by measuring at two nearby regions of the structure, provided that there is a certain permittivity difference between the materials behind the region of interest. The subtraction of these two measurements will enhance the pulse reflected at the end of the structure, including the creeping waves traveling around the rebar cross section, and will cancel the permanent reflections produced by the measurement system and the finite geometry of the concrete structure.

The transfer function $H(\omega)=S_{11}(\omega)$ is Fourier transformed to the time domain, and the resulting impulse responses for the concrete samples with the cover depth $\mathrm{C}$ are shown in Fig. 10. As it was observed in the through-transmission setup, a progressive displacement and attenuation of the main peak are observed due to the increase in the corrosion amount. Given the round-trip feature of the monostatic-reflection setup propagation, the main pulse sign is inverted with respect to the sign of the pulses in Fig. 9.

\section{E. Experiment 3: Bistatic Setup}

A bistatic setup combining the sensitivity of the throughtransmission setup and the field convenience of the monostaticreflection setup is tested in this section. The interest is focused on the transmission parameter $\mathrm{S}_{12}\left(=\mathrm{S}_{21}\right)$. The trans-

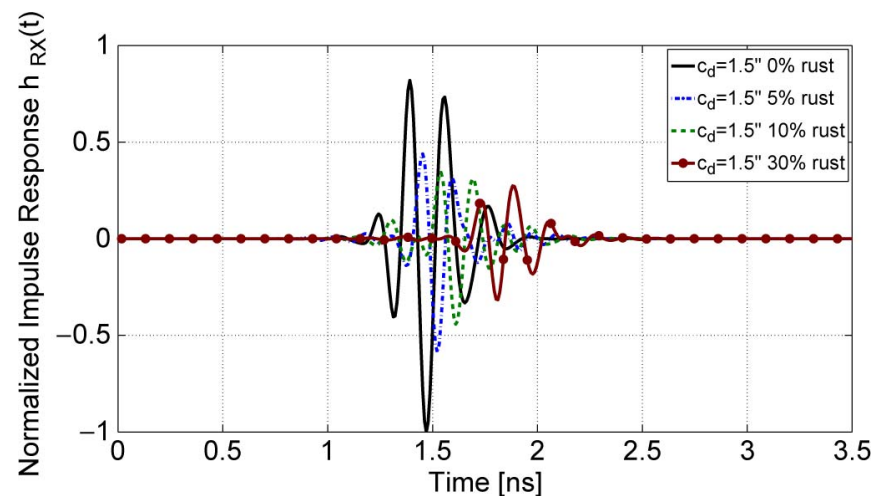

Fig. 11. Measured impulse response $h(t)$ normalized with respect to the noncorroded measurement, using a bistatic setup for the cover depth B and all the corrosion amounts from $0 \%$ to $30 \%$.

fer function $H(\omega)=S_{12}(\omega)$ is Fourier transformed to the time domain, and the resulting impulse responses for the concrete samples with the cover depth B are shown in Fig. 11. Again, the mean propagation delay of the main pulses increases and their attenuation decreases for increasing corrosion amounts. As it was observed in the monostatic-reflection setup measurement results, the main pulse sign is inverted with respect to the sign of the pulses in Fig. 9, due to the round-trip feature of the bistatic setup propagation.

Although the three antenna setups presented in this section achieve similar results, the bistatic setup is considered the most ideal one due to its practical advantages for the in situ inspection of concrete structures.

Finally, the quality factors, energy $\mathcal{E}$ and mean propagation delay $\tau$, are calculated for the bistatic setup according to (8) and (9), based on the experimental measurements on all the 12 reinforced concrete samples representing 3 concrete depths and 4 corrosion amounts. These parameters are calculated from the normalized impulse response with respect to the noncorroded case, which implies that the energy $\mathcal{E}$ and mean propagation delay $\tau$ are relative parameters with respect to the energy and the mean propagation delay of the noncorroded case. As plotted in Fig. 12, these factors represent a quantitative evaluation of the corrosion effects in the propagated field. Each corrosion amount is related to a certain combination of $\mathcal{E}$ and $\tau$, which determines the corrosion phase of the concrete structure. From the figure, an exponential decrease of $\mathcal{E}$ is observed as the corrosion amount increases (i.e., as the corrosion phase increases), reaching a saturation level at the higher corrosion amount, while $\tau$ increases approximately linearly with the corrosion amount. Therefore, for instance, from the results of the concrete samples with the cover depth B shown in Fig. 12, for a quality factor combination of $\mathcal{E}=0.45$ and $\tau=1.57 \mathrm{~ns}$, the system tells that the concrete structure is in a phase $2-3$ of the corrosion process (5\% rust), whereas when the quality combination is $\mathcal{E}=0.10$ and $\tau=1.80 \mathrm{~ns}$, the measurement system estimates that the concrete structure is in a phase 6 of the corrosion process. As the relation between energy, delay, and corrosion is given in relative terms, this relationship should be maintained even when the concrete mix design varies or corrosion is caused by other means. 


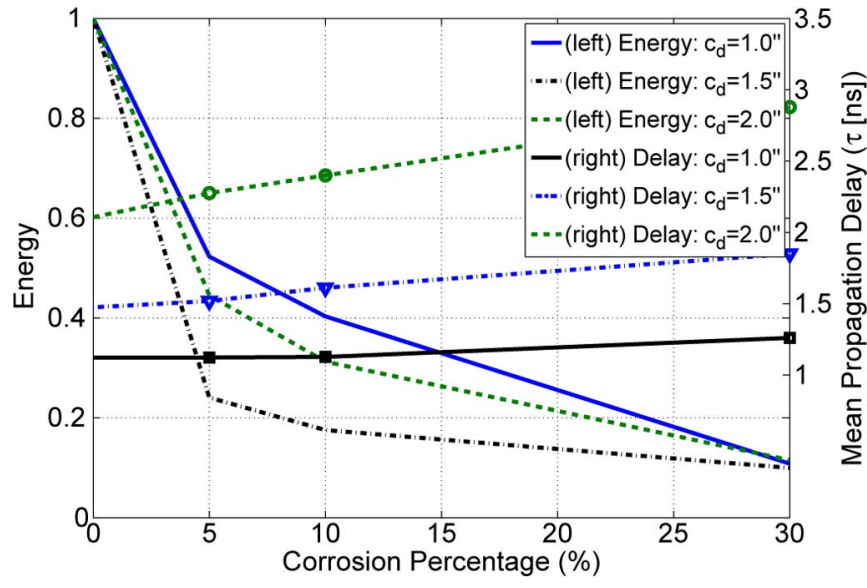

Fig. 12. Measured energy $(\mathcal{E})$ and mean propagation delay $(\tau)$ of the propagated field for each case in the bistatic arrangement.

\section{CONCLUSION}

This paper has presented analytical and experimental studies of the electromagnetic signature of corrosion in reinforced concrete structures for the purpose of developing an NDE technology for corrosion detection. First, the effects of the corrosion on the scattered field upon microwave illumination are analyzed. Second, first-order quality factors, energy and mean propagation delay, are identified as the optimal measurable parameters of the corrosion signature that contain the most information about the corrosion effects. Finally, experimental validation is performed on 12 reinforced concrete samples fabricated in this study, which involve four different corrosion amounts related to the different phases of the corrosion process and three different concrete cover depths. For the experiments, low profile UWB antennas (3-12 GHz) with a smooth impulse response and low level secondary lobes are fabricated.

From the analysis and experimental validation, the following points are concluded.

1) The two identified quality factors, the energy and the mean propagation delay, can detect steel rebar corrosion inside concrete and furthermore quantitatively evaluate the corrosion amount, being able to determine in which phase of the corrosion process the reinforced concrete structure is. The energy decreases exponentially and the mean propagation delay increases linearly as the corrosion amount in the rebar increases.

2) The three antenna setups tested in this study, the throughtransmission, monostatic-reflection, and bistatic setups, all achieved similar measurement results. From the practical point of view, the bistatic setup is recommended for in situ inspection of concrete structures.

3) Concrete specimens with three different cover depths are measured in this study. Although the measurement system provides sufficient sensitivity for all the three cases, the sensitivity performance increases for thicker cover depths due to the selected frequency bandwidth.

Providing insightful information about the electromagnetic signature of corrosion, this study lays a foundation for future development of a tomographic array for microwave imaging and quantitative NDE of rebar corrosion in concrete structures. The availability of such an inspection technology will make significant contributions to the safety and sustainability of concrete structures.

\section{REFERENCES}

[1] I. Rapoport, "Corroded steel reinforcing bars likely culprit," Daily Commercial News and Construction Record, 2006.

[2] G. H. Koch, M. Brongers, N. Thompson, Y. P. Virmani, and J. Payer, "Corrosion cost and preventive strategies in the United States," Federal Highway Administration, Washington, DC, Tech. Rep. FHWA-RD-01$156,2001$.

[3] P. Vassie, The Half-Cell Potential Method of Locating Corroding Reinforcement in Concrete Structures. Crowthorne, U.K.: Transp. Road Res. Lab., 1991.

[4] S. Feliu, J. Gonzales, and C. Andrade, "Multiple-electrode method for estimating the polarisation resistance in large structures," J. Appl. Electrochem., vol. 26, no. 3, pp. 305-309, Mar. 1996.

[5] N. Gucunski, C. Rascoe, R. Parrillo, and R. Roberts, "Comparative study of bridge deck condition assessment by high frequency GPR," in Proc. Symp. NDE/NDT Highways Bridges, SMT, 2008, pp. 58-66.

[6] R. Arndt and F. Jalinoos, "NDE for corrosion detection in reinforced concrete structures-A benchmark approach," in Proc. Int. Symp. NDTCE, Nantes, France, Jun. 30, 2009, pp. 1-6.

[7] D. Chen and S. Mahadevan, "Chloride-induced reinforcement corrosion and concrete cracking simulation," Cement Concrete Composites, vol. 30, no. 3, pp. 227-238, Mar. 2008.

[8] R. E. Weyers, M. G. Fitch, E. P. Larsen, I. L. Al-Qadi, W. P. Chemberlin, and P. Hoffman, "Concrete bridge protection and rehabilitation: Chemical and physical techniques," in Proc. SHRP-S-668, Nat. Res. Council, Washington, DC, 1994, p. 90.

[9] Tech. Rep. Concrete in Practice: What, Why and How? CIP 25Corrosion of Steel in Concrete, Silver Spring, MD, 1995. [Online]. Available: www.nrmca.org

[10] A. Schmidt, "Physiochemical changes in London clay adjacent to cast iron pipes," Ph.D. dissertation, Univ. Birmingham, Birmingham, U.K., 2008.

[11] J. F. Aurand, "Measurements of short-pulse propagation through concrete walls," in Proc. 3rd Int. Conf. Ultra Wide Band Short-Pulse Electromagn., Albuquerque, NM, 1996, p. 8.

[12] R. F. Harrington, "Time-harmonic electromagnetic fields," in The IEEE Press Series on Electromagnetic Wave Theory, I. P. E. Board, Ed. Piscataway, NJ: IEEE Press, 2001.

[13] M. Abramowitz and I. A. Stegun, Handbook of Mathematical Functions with Formulas, Graphs, and Mathematical Tables. New York: Dover, 1970.

[14] A. F. Oskooi, D. Roundy, M. Ibanescu, P. Bermel, J. Joannopoulos, and S. G. Johnson, "MEEP: A flexible free-software package for electromagnetic simulations by the FDTD method," Comput. Phys. Commun., vol. 181, no. 3, pp. 687-702, Mar. 2010.

[15] H. Rhim and O. Büyüköztürk, "Electromagnetic properties of concrete at microwave frequency range," ACI Mater. J., vol. 95, no. 3, pp. 262-271, May 1998

[16] M. D. Belrhiti, S. Bri, A. Nakheli, M. Haddad, and A. Mamouni, "Complex permittivity measurement for dielectric materials at microwave frequencies using rectangular waveguide," Eur. J. Sci. Res., vol. 49, no. 2, pp. 234-248, 2011.

[17] D. Hughes, N. Wang, T. Case, K. Donnell, and R. Zoughi, "Microwave nondestructive detection of corrosion under thin paint and primer in aluminum panels," Subsurf. Sens. Technol. Appl., vol. 2, no. 4, pp. 435-451, Oct. 2001.

[18] G. Roqueta, L. Jofre, J. Romeu, and S. Blanch, "Microwave time domain reflection imaging of steel fiber distribution on reinforced concrete," IEEE Trans. Instrum. Meas., vol. 60, no. 12, pp. 3913-3922, Dec. 2011.

[19] A. F. Molisch, Wireless Communications, J. W. Sons, Ed. Piscataway, NJ: IEEE Press, 2005.

[20] A. C. Gangadharan, Leak Prevention and Corrective Action Technology for Underground Storage Tanks. Park Ridge, NJ: Noyes Data Corp., 1998, ser. Pollution Technol. Rev., no. 153.

[21] H. Schantz, The Art and Science of Ultrawideband Antenas. Norwood, MA: Artech House, 2005. 


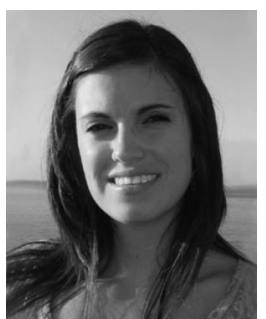

Gemma Roqueta ( $\left.\mathrm{S}^{\prime} 08\right)$ was born in Llagostera (Girona), Spain, in 1983. She received the degree in telecommunication engineering from the Technical University of Catalonia (UPC), Barcelona, Spain, in 2007. She is currently working toward the Ph.D. degree at the UPC, where she is working on the development of wideband microwave imaging applications for nondestructive quality testing methods for civil structures.

In 2006, she was involved in Body Area Networks research as a foreign student at the Université Catholique de Louvain, Louvain-la-Neuve, Belgium. From August 2010 to December 2010, she has been a Visiting Scholar at the Civil and Environmental Engineering Department, Henry Samueli School of Engineering, University of California, Irvine, under the FPU fellowship program, where she worked on antennas and electromagnetic imaging and visualization systems for civil structures with corrosion. Her research interests include indoor and outdoor propagation, wideband microwave imaging for the nondestructive testing of civil engineering structures, spiralometric discrimination of embedded objects, and ultra-wide-band antennas.

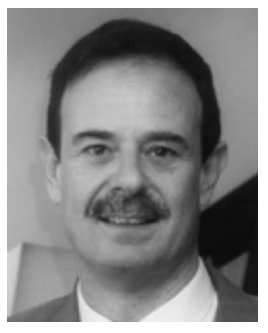

Lluís Jofre (S'79-M'83-SM'07-F'10) was born in Canet de Mar, Spain, in 1956. He received the M.Sc. (Ing.) and Ph.D. (Dr. Ing.) degrees in electrical engineering (telecommunication engineering), from the Technical University of Catalonia (UPC), Barcelona, Spain, in 1978 and 1982, respectively.

From 1979 to 1980, he was a Research Assistant with the Electrophysics Group, UPC, where he worked on the analysis and near field measurement of antennas and scatterers. From 1981 to 1982 , he was with the École Supérieure d'Electricité, Paris, France, where he was involved in microwave antenna design and imaging techniques for medical and industrial applications. Since 1982, he has been with the Communications Department, Telecomunication Engineering School, UPC, as an Associate Professor first and, then, as a Full Professor since 1989. From 1986 to 1987, he was a Visiting Fulbright Scholar at the Georgia Institute of Technology, Atlanta, where he worked on antennas and electromagnetic imaging and visualization. From 1989 to 1994, he was the Director of the Telecommunication Engineering School, UPC, and from 1994 to 2000, he was the UPC Vice-Rector for Academic Planning. From 2000 to 2001, he was a Visiting Professor at the Electrical and Computer Engineering Department, Henry Samueli School of Engineering, University of California, Irvine. From 2002 to 2004, he was the Director of the Catalan Research Foundation, and since 2003, he has been the Director of the UPC-Telefonica Chair and Director of the Promoting Engineering Catalan Program "EnginyCAT." He has published more than 100 scientific and technical papers, reports, and chapters in specialized volumes. His research interests include antennas, electromagnetic scattering and imaging, and system miniaturization for wireless and sensing industrial and bioapplications.

Dr. Jofre is a member of different Higher Education Evaluation Agencies at Spanish and European levels. From December 2011, he is the General Director of Universities in the Economy and Knowledge Council of the Catalan Government. At international level, he is a fellow of the IEEE Society.

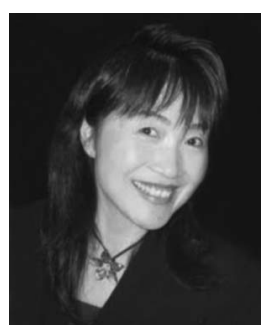

Maria Q. Feng received the Ph.D. degree in mechanical engineering from the University of Tokyo, Tokyo, Japan, in 1992.

She joined the University of California, Irvine (UCI), Irvine, in 1992, where she has been Professor of Civil and Environmental Engineering until 2011, with a joint appointment at the Department of Electrical Engineering and Computer Science. During this period, she has been the Founding Director of the Center for Advanced Monitoring and Damage Detection at UCI. Since January 2012, she joined the Columbia University as Renwick Professor at the Department of Civil Engineering and Engineering Mechanics. Her research interests are primarily in safety and security of civil infrastructure systems, and military armor protective systems, focusing on innovative and interdisciplinary science and technology for sensors, health monitoring, and damage assessment. She has published near 350 journal and conference proceeding papers on these subjects.

Dr. Feng was the recipient of numerous awards and honors, including the following: 1) CAREER Award by the National Science Foundation; 2) Alfred Noble Prize jointly by the American Society of Civil Engineers (ASCE), the American Society of Mechanical Engineering, the Institute of Electrical and Electronics Engineers, the American Institute of Mining, Metallurgical, and Petroleum Engineers (AIME), and Western Society of Engineers; 3) Collingwood Prize by ASCE; 4) Charles Pankow Finalist Award for Innovation by ASCECivil Engineering Research Foundation; 5) Walter L. Huber Civil Engineering Research Prize by ASCE; 6) Best Presentation Award by Society of Instrument and Control Engineers, Japan; and 7) Fariborz Maseeh Best Faculty Research Award by UCI. She is a fellow of ASCE. 